\title{
From lists of behaviour change techniques (BCTs) to structured hierarchies: Comparison of two methods of developing a hierarchy of BCTs
}

\author{
James Cane ${ }^{\prime *}$, Michelle Richardson ${ }^{2}$, Marie Johnston ${ }^{3}$, \\ Ruhina Ladha ${ }^{4}$ and Susan Michie ${ }^{4}$ \\ 'School of Psychology, Keynes College, University of Kent, Canterbury, UK \\ ${ }^{2}$ Department of Health Services and Population Research, King's College London, UK \\ ${ }^{3}$ Institute of Applied Health Sciences, College of Life Sciences and Medicine, University \\ of Aberdeen, UK \\ ${ }^{4}$ Research Department of Clinical, Education, and Health Psychology, University \\ College London, UK
}

Objectives. Behaviour change technique (BCT) Taxonomy $\mathrm{vl}$ is a hierarchically
grouped, consensus-based taxonomy of $93 \mathrm{BCTs}$ for reporting intervention content. To
enhance the use and understanding of BCTs, the aims of the present study were to (I)
quantitatively examine the 'bottom-up' hierarchical structure of Taxonomy vI, ( 2 )
identify whether BCTs can be reliably mapped to theoretical domains using a 'top-down'
theoretically driven approach, and (3) identify any overlap between the 'bottom-up' and
'top-down' groupings.

Methods and design. The 'bottom-up' structure was examined for higher-order groupings using a dendrogram derived from hierarchical cluster analysis. For the theory-based 'top-down' structure, 18 experts sorted BCTs into 14 theoretical domains. Discriminant Content Validity was used to identify groupings, and chi-square tests and Pearson's residuals were used to examine the overlap between groupings.

Results. Behaviour change techniques relating to 'Reward and Punishment' and 'Cues and Cue Responses' were perceived as markedly different to other BCTs. Fifty-nine of the BCTs were reliably allocated to I 2 of the $I 4$ theoretical domains; 47 were significant and 12 were of borderline significance. Thirty-four of 208 'bottom-up' $\times$ 'top-down' pairings showed greater overlap than expected by chance. However, only six combinations achieved satisfactory evidence of similarity.

Conclusions. The moderate overlap between the groupings indicates some tendency to implicitly conceptualize BCTs in terms of the same theoretical domains. Understanding the nature of the overlap will aid the conceptualization of BCTs in terms of theory and application. Further research into different methods of developing a hierarchical taxonomic structure of BCTs for international, interdisciplinary work is now required.

\footnotetext{
*Correspondence should be addressed to James Cane, School of Psychology, Keynes College, University of Kent, Canterbury CT2 7NP, UK (email: j.e.cane@kent.ac.uk).
} 


\section{Statement of contribution}

What is already known on this subject?

- Behaviour change interventions are effective in improving health care and health outcomes.

- The 'active' components of these interventions are behaviour change techniques and over 93 have been identified.

- Taxonomies of behaviour change techniques require structure to enable potential applications.

\section{What does this study add?}

- This study identifies groups of BCTs to aid the recall of BCTs for intervention coding and design.

- It compares two methods of grouping - 'bottom-up' and theory-based 'top-down' - and finds a moderate overlap.

- Building on identified BCT groups, it examines relationships between theoretical domains and BCTs.

Preventable behaviours, such as smoking, alcohol consumption, and overeating, have been identified as a major cause of mortality and morbidity in the Western world (Mokdad, Marks, Stroup, \& Gerberding, 2004; Parkin, Boyd, \& Walker, 2011). Interventions to change such behaviours are key to improving population health (Michie \& Johnston, 2012). Such interventions are usually complex, comprising several interacting components. To develop more effective interventions, it is necessary to investigate the combinations of components that are most effective in changing a particular behaviour. There have been calls and guidelines from international consortia such as Consolidated Standards of Reporting Trials (CONSORT) and the UK Medical Research Council (MRC) for improved methods of identifying and reporting the components of interventions (Boutron, Moher, Altman, Schulz, \& Ravaud, 2008; Craig et al., 2008). In response to these calls, researchers have begun to specify the contents, or 'active ingredients', of interventions in the form of behaviour change techniques (BCTs, see Abraham \& Michie, 2008; Michie, Abraham, et al., 2011; Michie et al., 2013; Michie \& Johnston, 2013).

Behaviour change techniques are the smallest components of behaviour change interventions that on their own have the potential to change behaviour. They are observable and replicable and can be used alone or in combination with other BCTs (Michie et al., 2013). An example of a BCT would be 'self-monitoring of behaviour', defined as 'Instruct self-recording of specified behaviour/s (with or without associated thoughts, emotions, situations) as part of a behaviour change strategy', for example, keeping a daily diary of food consumption. Lists of BCTs have been developed in relation to both specific behaviours (e.g., physical activity, healthy eating, smoking, alcohol use, condom use, and changing professional behaviour) and for use across behavioural domains (Abraham, Good, Warren, Huedo-Medina, \& Johnson, 2011; Ivers et al., 2012; Lorencatto, West, \& Michie, 2012; Michie, Hyder, Walia, \& West, 2011; Michie, Whittington, et al., 2012; Michie, Abraham, Whittington, McAteer, \& Gupta, 2009; West, Walia, Hyder, Shahab, \& Michie, 2010). The application of such classification systems is growing rapidly (e.g., Araújo-Soares, McIntyre, MacLennan, \& Sniehotta, 2009; Chadwick \& Benelam, 2013; Dombrowski et al., 2012; Free et al., 2013; Michie, Free, \& West, 2012).

Recent work has synthesized existing BCT taxonomies into a single comprehensive, cross-context, overarching BCT taxonomy: BCT Taxonomy v1 (Michie et al., 2013; Michie, Johnston, et al., 2012). This BCT taxonomy was developed through a series of consensus exercises (including a Delphi exercise, feedback from an international advisory board and coding exercises) involving over 50 behaviour change experts from a variety of disciplines and countries. It comprises 93 BCTs (see Appendix S1 for the version used in 
the present study) which have been evaluated on (1) whether they conformed to a definition of a BCT, (2) their clarity as an individual BCT, and (3) their distinctiveness (i.e., whether there was any overlap and redundancy with other BCTs).

To increase the usability and speed of recall of BCTs, BCT Taxonomy v1 was organized hierarchically using an open-sort task and hierarchical cluster analysis (HCA). BCTs were grouped using an inductive 'bottom-up' method based on the similarity of their active ingredients. This process yielded 16 distinct sets of BCTs, as follows (with number of BCTs in parentheses): Scheduled Consequences (10), Reward/Threat (7), Repetition/Replacement (7), Antecedents (4), Associations (8), Covert Learning (3), Natural Consequences (6), Feedback \& Monitoring (5), Goals \& Planning (9), Social Support (3), Comparison of Behaviour (3), Self-belief (4), Comparison of Outcomes (3), Identity (5), Shaping Knowledge (4), and Adjunctive (4). In contrast to previous BCTs groupings that are based on judgements made by the study authors (see Dixon \& Johnston, 2012; Michie, Churchill, \& West, 2011; Michie, Hyder, et al., 2011), these groupings were identified empirically, by allowing experts (from a range of disciplines) to individually create groups that were then aggregated using empirical techniques. This approach yielded a hierarchical structure, where groupings of BCTs were nested within higher-order groupings.

The usefulness of BCT Taxonomy $\mathrm{v} 1$ for understanding and designing interventions would be enhanced if an agreed method were developed to facilitate the choice of BCTs by identifying links between particular BCTs and theoretical constructs. Theories specify key relationships that are important in the process of behaviour change and describe the conditions where behaviour change is most likely to occur (Michie \& Johnston, 2012). The importance of understanding the theoretical underpinnings of BCTs has been highlighted in previous research suggesting that having a theoretical basis to an intervention enhances its effectiveness (Dombrowski et al., 2012; Michie et al., 2009; Taylor, Conner, \& Lawton, 2012; Webb, Joseph, Yardley, \& Michie, 2010). Furthermore, a recent meta-analysis found that behaviour change interventions often do not have a clear theoretical foundation from which they are designed - of 235 implementation studies, only $22.5 \%$ were judged to have used theories of behaviour change (Davies, Walker, \& Grimshaw, 2010) and the majority of these gave no clear explanation for why the selected theories had been used. There is therefore a clear need for improving methods for applying theory to intervention design and our understanding of how BCTs exert their influences. Grouping BCTs by theory would help guide understanding of the functional relationships between BCTs, the underlying mechanisms through which they exert their effects and the most effective ways in which BCTs can be applied.

In the light of the number of BCTs reported in the taxonomy and the large number of behaviour change theories (see Hobbs, Campbell, Hildon, \& Michie, 2011), grouping by individual theories or theoretical constructs is impractical. An alternative is to group by broader domains (e.g., knowledge, skills) from a framework that groups theoretical constructs, such as the Theoretical Domains Framework (TDF; Cane, O'Connor, \& Michie, 2012; Michie et al., 2005). The TDF is an integrative framework of theories of behaviour change that was originally developed by 18 psychological theorists in collaboration with 16 health service researchers and 30 health psychologists (Michie et al., 2005). It synthesizes behaviour change theories and constructs to make theory more accessible to, and usable by, a range of disciplines and theoretical orientations. The first version of the TDF contains 12 theoretical domains synthesized from 128 theoretical constructs related to behaviour change.

The TDF has been used by research teams across several countries and health care systems to investigate implementation problems and inform interventions to change 
professional practice (see Amemori, Korhonen, Kinnunen, Michie, \& Murtomaa, 2011; Beenstock et al., 2012; Bussières, Patey, Francis, Sales, \& Grimshaw, 2012; Dyson, Lawton, Jackson, \& Cheater, 2011; Francis, Stockton, et al., 2009; Francis, Tinmouth, et al., 2009; French et al., 2011; McKenzie et al., 2008, 2010; Patey, Islam, Francis, Bryson, \& Grimshaw, 2012). The TDF has been validated using two sort tasks (see Cane et al., 2012), producing a refined TDF containing 87 theoretical constructs relevant to behaviour change categorized across 14 domains: Knowledge, Skills, Social/Professional Role and Identity, Beliefs about Capabilities, Optimism, Beliefs about Consequences, Reinforcement, Intentions, Goals, Memory, Attention and Decision Processes, Environmental Context and Resources, Social Influences, and Emotion and Behavioural Regulation. A previous attempt to map 35 BCTs to 11 theoretical domains from the original TDF showed good reliability across four researchers, with $71 \%$ agreement over the 385 possible mappings (Michie, Johnston, Francis, Hardeman, \& Eccles, 2008). Building on this work, we aim to link BCTs from the more comprehensive BCT Taxonomy v1 to the refined TDF, using a number of international experts in behaviour change.

In summary, this study aims to

1. investigate the hierarchical structure of the groupings of the taxonomy, which were obtained from an inductive 'bottom-up' approach, using a dendrogram derived from quantitative clustering methods.

2. identify whether and to what extent the taxonomy can be reliably grouped using a deductive, 'top-down' theory-based approach into the 14 theory-based domains of the revised TDF.

3. examine similarities and differences in the groupings that emerge using these two methods of developing a hierarchical structure.

\section{Method}

\section{Examining the hierarchical structure of BCT Taxonomy vI using an inductive ('bottom-up') approach}

For full details of the participants, methods, and procedure, see Michie et al. (2013).

\section{Participants}

Eighteen behaviour change experts who took part in the development of BCT Taxonomy v1 (see Table 1 for expert demographic information).

\section{Procedure}

The BCT groupings found in the taxonomy were developed using an open-sort grouping task delivered via an online computer program. Participants were asked to sort the list of BCTs into groups (up to a maximum of 24) of their choice and label the groups. Instructions guided the experts to 'group together BCTs which have similar active ingredients, i.e., by the mechanism of change, NOT the mode of delivery'. Individual expert's BCT groups were aggregated, and HCA was conducted to identify the optimal number of groupings (clusters) for the BCTs (between 2 and 20 groupings). The data from the open-sort task indicated 16 groupings as the best fit for the data, giving greatest stability as indexed by the Figure-of-Merit (FOM) statistic (see Michie et al., 2013), and the hierarchical structure was illustrated using a dendrogram (see Figure 1). Using the 
Table I. Demographic information for open- and closed-sort tasks

\begin{tabular}{lll}
\hline Sort task & Open 'bottom-up' & Closed 'top-down' \\
\hline Age & & \\
Mean & 43.94 & 40.83 \\
SD & 13.58 & 10.47 \\
Range & $27-67$ & $24-63$ \\
Gender - no. of participants & & 15 \\
Women & 8 & 3 \\
Men & 10 & \\
Country - no. of participants & & 1 \\
Australia & 2 & 1 \\
Italy & & 1 \\
The Netherlands & & 5 \\
New Zealand & 16 & 10 \\
UK & & \\
USA & & \\
\hline
\end{tabular}

dendrogram derived from the HCA in Michie et al. (2013), we assessed the hierarchical structure of the BCT groupings. The distance between the groupings at each split is indicated by the 'height' on the $Y$-axis of the dendrogram, with greater height values indicating greater distance and less similarity between the groups, and lower height values indicating less distance and greater similarity between the groups.

\section{Examining the hierarchical structure of the taxonomy using a deductive, theoretically based ('top-down') approach}

Participants

Twenty-five individuals were invited to take part in the closed-sort task. Participants were eligible to take part if they had (1) experience in designing interventions that specifically used BCTs, (2) experience in writing manuals or protocols of behaviour change interventions, or (3) undertaken a narrative or systematic review of behaviour change literature. Participants were recruited via announcements through university networks and scientific societies' mailing lists - the Society of Behavioural Medicine, the APA Health Division, and the Society for Academic Primary Care. Eighteen people (72\%) met the eligibility criteria and all who were eligible consented to complete the task (see Table 1 for demographic information). There was no overlap in participants between the 'bottom-up' and 'top-down' sort tasks. The sample size for the closed-sort task was based on estimates given for content-validation exercises, with two to 24 participants being shown to be sufficient (see Dixon, Johnston, McQueen, \& Court-Brown, 2008; Dixon, Pollard, \& Johnston, 2007; Lynn, 1986; Waltz, Strickland, \& Lenz, 1991) and more than five participants reducing the influence of rater outliers (Carmines \& Zeller, 1979).

\section{Materials}

Participants sorted BCTs into the 14 domains specified in the revised version of the TDF (Cane et al., 2012): Knowledge, Skills, Social/Professional Role and Identity, Beliefs about Capabilities, Optimism, Beliefs about Consequences, Reinforcement, Intentions, Goals, 


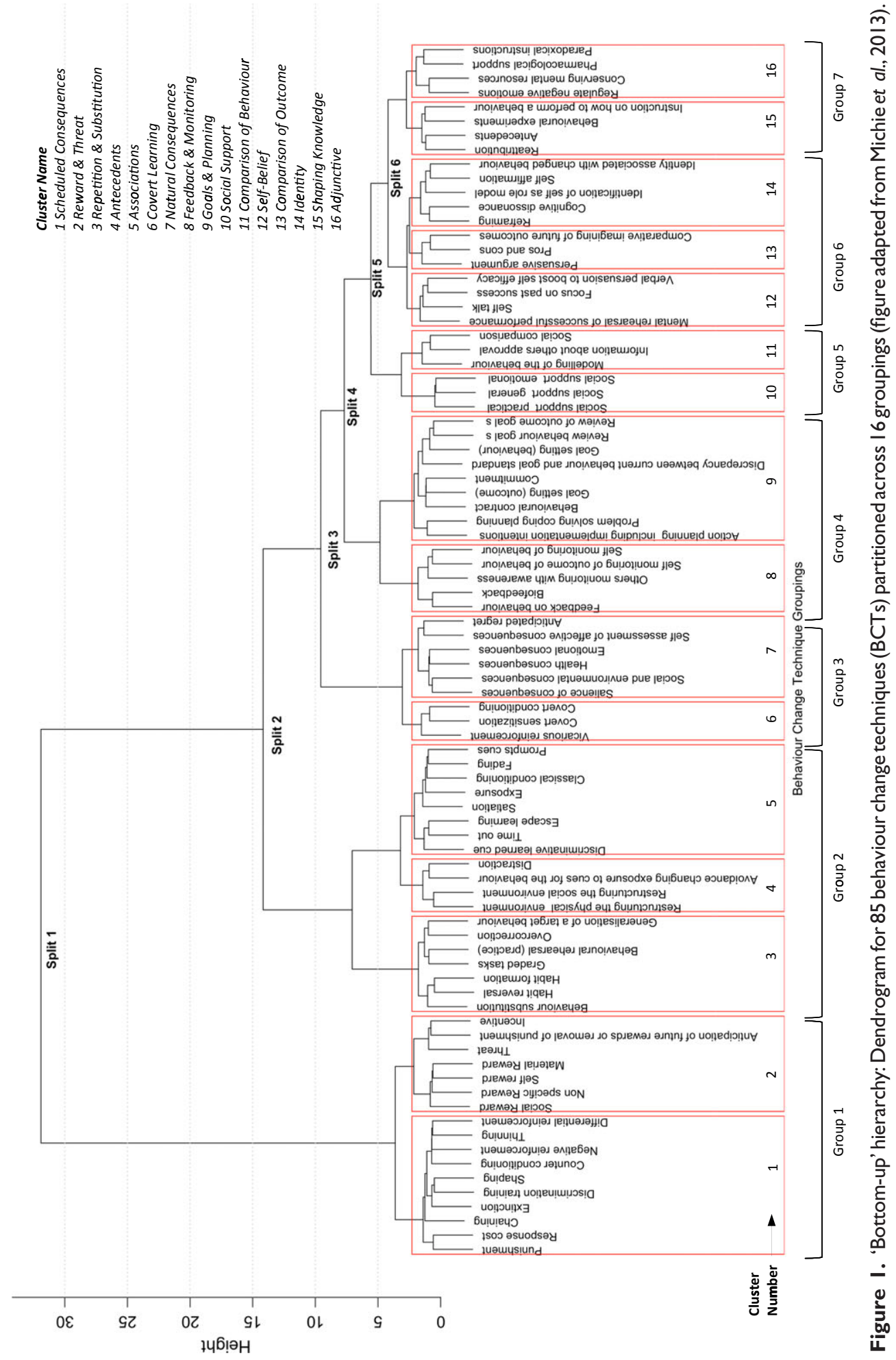


Memory, Attention and Decision Processes, Environmental Context and Resources, Social Influences, and Emotion and Behavioural Regulation (see Appendix S2).

The closed-sort task was delivered via a Word document, comprising labels and definitions of the 14 theoretical domains and of the $87^{1}$ BCTs from BCT Taxonomy v1 (see Appendix S1), which were randomly ordered (Michie et al., 2013). Participants were required to indicate which domain was most relevant for each $\mathrm{BCT}$ and give a confidence rating for their allocation (see Appendix S3 for example).

\section{Procedure}

Invitations included a brief overview of the study and participation consent form. Consenting participants were given detailed instructions on how to complete the task and were asked to provide demographic information (including age, gender, and nationality) and to rate their expertise in behaviour change theory and in delivering behaviour change interventions on a 5-point scale $(1=$ A great deal, $2=$ quite a bit, $3=$ some, $4=$ a little , 5 = none).

Participants were asked to allocate each of the 87 BCTs to one or more of the 14 theoretical domain(s), giving a confidence rating for each allocation (from $1=$ not at all confident to $10=$ extremely confident). After assigning all BCTs, participants were asked to review their BCT allocations and to revise any allocations if they wanted to. There was no time limit for the tasks, and participants were debriefed about the study on completion.

\section{Data analysis}

Mean confidence ratings for each BCT $\times$ domain pairing were calculated and analysed using Discriminant Content Validity methods (DCV; see Dixon et al., 2008). BCT $\times$ domain pairings that had no confidence rating from individual participants (i.e., that BCT was not allocated to that domain by that participant) were scored 0 and entered into the mean score for that pairing. A series of one-sample $t$-tests compared the mean confidence ratings for the assignment of BCTs to a value of 0 . This established the extent to which BCTs were related to each domain. In cases where no experts allocated a BCT to a specific domain (i.e., all scores for a BCT $\times$ domain pairing were 0 ), the $\mathrm{BCT} \times$ domain pairings were excluded from $t$-test analyses.

Behaviour change techniques were considered to be reliably allocated to a domain if their mean confidence ratings were significantly greater than zero $(p<.05)$ after Hochberg's correction (Hochberg, 1988; applied using the p.adjust function in R [R Development Core Team, 2008]). This was used to control for the family-wise error rate given the large number of tests used and provided a suitable criterion for inclusion and exclusion of BCTs to a particular domain, over and above the use of a subjective cut-off value. Hochberg's correction also provides a conservative $p$-value that makes it less likely that a BCT $\times$ domain pairing achieving low confidence ratings across the majority of participants will achieve significance. The agreement of BCT allocation across participants was analysed using a two-way intraclass correlation coefficient (ICC) within each domain.

\footnotetext{
${ }^{I}$ The closed-sort task was conducted on an earlier version of the taxonomy containing 87 BCTs (see Appendix SI) and the open-sort task an even earlier version containing 85 BCTs. Both were conducted whilst the BCT Taxonomy vl was in development - the published version of BCT Taxonomy vl contains 93 BCTs (see Michie et al., 2013).
} 


\section{Comparison of sort task groupings}

Two types of comparisons were made between the 'bottom-up' groupings and the 'top-down' TDF-derived groupings - comparison between the theoretically derived 'top-down' groupings and (1) the higher-order strategy groupings used in the 'bottom-up' sort task, and (2) the final groupings of the 'bottom-up' sort task. To test the possibility of overlap between groupings derived from using 'bottom-up' and 'top-down' methods, Pearson's chi-square test was adopted. To adjust for potential inaccuracy of the $p$-value estimation (resulting from the number of cells that had expected frequencies $<1$ ), Monte Carlo simulation (using 2000 replications) was used. Pearson's residual values ((observed - expected)/sqrt (expected)) were used to quantify the extent of overlap between individual BCT Taxonomy $\mathrm{v} 1$ group $\times$ TDF domain pairings resulting from the 'bottom-up' and 'top-down' methods. Positive values indicate that the observed overlap in BCT assignment between the BCT Taxonomy group and TDF domain is greater than expected by chance, whereas negative values indicate that it is less than expected.

\section{Results}

\section{Grouping of BCTs using an inductive ('bottom-up') approach}

Within the reported 16-group open-sort solution of the taxonomy, there are six points at which groups of BCTs split into groups containing similar BCTs (creating seven split groups, that is, higher-order strategy groupings; see Figure 1 and Table S1). These groups themselves contain more subtle distinct groupings as detailed in the BCT Taxonomy v1. The first split is at 'split 1' (height $=31.78$ ), where the body of BCTs split into two groups, the grouping to the left containing the groupings of 'Scheduled consequences' and 'Reward and Threat' that involve BCTs relating to the anticipation of a direct reward or punishment (e.g., social reward, negative reinforcement, extinction). The next split, 'split 2 ' (height $=14.16$ ), reveals three groupings to the left of the remaining BCTs: 'Repetition and Substitution', 'Antecedents', and 'Associations' comprising BCTs relating to cues and cue responses. From split 3 onwards, the distance between the groupings is markedly smaller (height $<10$ ), indicating that the groupings formed are less distinct from each other. At split 3 (height =9.56), BCTs from the groupings 'Covert Learning' and 'Natural Consequences' are separated off from the remaining groupings. At split 4 (height = 7.69), the split includes the groupings 'Feedback and Monitoring', and 'Goals and Planning' and BCTs relating to goals, planning and feedback. At split 5 (height $=5.55$ ), the split includes the groupings 'Social Support' and 'Comparison of Behaviour' and BCTs related to social factors. The final split occurs at split 6 (height $=4.18)$, where the groupings 'Self-Belief', 'Comparison of Outcome', and 'Identity' (BCTs relating to the self and identity) are separated from the groupings of 'Shaping Knowledge' and 'Regulation' (BCTs relating to knowledge and regulation).

\section{Grouping BCTs using theoretical domains (deductive, 'top-down' approach)}

Participants for the closed-sort task reported moderately high levels of expertise in behaviour change theory $(M=3.17, S D=0.71)$ and in delivering behaviour change interventions $(M=2.17, S D=1.38)$ as measured on 5-point scales (scores are reversed so higher score indicates more experience). This is not significantly different from the level of expertise reported by participants in the open-sort task from the taxonomy (behaviour change theory, $M=3.00, S D=0.88, t(34)=0.64, p>.1$; behaviour change interven- 
Table 2. Total number of behaviour change technique (BCT) allocations per domain in the closed-sort 'top-down' task

\begin{tabular}{lcc}
\hline Domain & $\begin{array}{c}\text { Number of BCTs } \\
\text { allocated }\end{array}$ & $\begin{array}{c}\text { Number of BCTs } \\
\text { allocated where } p<. I\end{array}$ \\
\hline Knowledge & 40 & 4 \\
Skills & 44 & 5 \\
Social/professional role and identity & 15 & 0 \\
Beliefs about capabilities & 46 & 2 \\
Optimism & 24 & 1 \\
Beliefs about consequences & 46 & 10 \\
Reinforcement & 45 & 17 \\
Intentions & 27 & 2 \\
Goals & 29 & 5 \\
Memory, attention and decision & 49 & 0 \\
Environmental context and resources & 42 & 5 \\
Social influences & 42 & 10 \\
Emotion & 44 & 4 \\
Behavioural regulation & 68 & 1 \\
\hline
\end{tabular}

tions, $M=2.42, S D=0.96, t(34)=0.63, p>.1)$. Whilst the age of participants did not differ significantly between the two sort tasks, $t(34)=0.77, p>.1$, the number of female and male participants did, $\chi^{2}(1)=4.33, p<.05$, as did the country of residence $\left(\chi^{2}=20.76 p<.001\right)^{2}$. This was an artefact of the selection process as there was no duplication of participants across the two sort tasks.

All TDF domains had BCTs allocated to them in the closed-sort task, with the number of BCTs allocated ranging from 15 for Social/Professional Role and Identity to 68 for Bebavioural Regulation (see Table 2). This allocation was reliable for 12 of the 14 domains, that is, BCTs were allocated consistently with high confidence across experts leading to $p<.05$ (see Table 2 for frequencies and Table 3 for confidence ratings, ICC values and related $p$-values). Within these domains, 59 (68\%) of the BCTs were considered to be reliably allocated, with a further 12 (14\%) BCTs having borderline statistical significance $(p>.05$ but $p<.1)$ and six being allocated to multiple domains (see Table 3). The domains, in order of number of BCT allocations obtaining statistical or marginal statistical significance, were (numbers of BCTs in brackets) Reinforcement (17), Beliefs about Consequences (10), Social Influences (10), Goals (6), Environmental Context and Resources (6), Skills (5), Emotion (5), Knowledge (4), Beliefs about Capabilities (2), Intentions (2), Optimism (1), and Bebavioural Regulation (1). Two domains, 'Social/Professional Role and Identity' and 'Memory, Attention and Decision Processes' had no BCTs significantly assigned to them. This indicates that whilst both of these domains had BCTs allocated to them during the sort process (15 and 49, respectively), experts did not consistently allocate or rate highly any of the BCTs to these two domains.

Of the 24 most commonly occurring BCTs (see Michie et al., 2013; emboldened in Table 3), 18 (75\%) were reliably mapped to seven of the theory domains, with further two (8\%) obtaining borderline statistical significance. These domains were (with number of

\footnotetext{
${ }^{2}$ Monte Carlo simulation using 2000 replicates was used to compute the $\mathrm{p}$-value given that a number of the expected cell values were $<I$.
} 
Table 3. Assignment of BCTs to the Theoretical Domains Framework domains in the closed-sort 'top-down' task (where $p<$. I)

\begin{tabular}{|c|c|c|c|c|c|}
\hline \multirow[b]{2}{*}{ Domain label and associated BCTs } & \multirow{2}{*}{$\begin{array}{l}\text { Mean } \\
\text { confidence } \\
\text { rating }\end{array}$} & \multirow{2}{*}{$\begin{array}{l}\text { Associated } \\
\text { probability }\end{array}$} & \multicolumn{2}{|c|}{$\begin{array}{c}95 \% \\
\text { confidence } \\
\text { intervals }\end{array}$} & \multirow{2}{*}{$\begin{array}{l}\text { Intraclass } \\
\text { correlation } \\
(p<.005)\end{array}$} \\
\hline & & & Lower & Upper & \\
\hline \multicolumn{6}{|l|}{ Knowledge } \\
\hline Health consequences & 6.06 & .001 & 3.80 & 8.32 & \\
\hline Biofeedback & $3.78^{\mathrm{a}}$ & .066 & 1.66 & 5.90 & .15 \\
\hline Antecedents & $3.72^{\mathrm{a}}$ & .051 & 1.71 & 5.74 & \\
\hline Feedback on behaviour & $3.67^{\mathrm{a}}$ & .057 & 1.65 & 5.68 & \\
\hline \multicolumn{6}{|l|}{ Skills } \\
\hline Graded tasks & 4.89 & .014 & 2.62 & 7.16 & \\
\hline Behavioural rehearsal/practice & 4.78 & .016 & 2.53 & 7.02 & .16 \\
\hline Habit reversal & 4.33 & .018 & 2.27 & 6.40 & \\
\hline Body changes & 4.06 & .020 & 2.08 & 6.03 & \\
\hline Habit formation & $4.33^{\mathrm{a}}$ & .091 & 1.57 & 5.88 & \\
\hline Social/professional role and identity & N/A & $\mathrm{N} / \mathrm{A}$ & N/A & N/A & .07 \\
\hline \multicolumn{6}{|l|}{ Beliefs about Capabilities } \\
\hline Verbal persuasion to boost self-efficacy ${ }^{b}$ & 5.11 & .015 & 2.72 & 7.50 & .11 \\
\hline Focus on past success & 4.33 & .040 & 2.07 & 6.60 & \\
\hline \multicolumn{6}{|l|}{ Optimism } \\
\hline Verbal persuasion to boost self-efficacy ${ }^{b}$ & 3.83 & .049 & 1.62 & 6.05 & .09 \\
\hline \multicolumn{6}{|l|}{ Beliefs about Consequences } \\
\hline Emotional consequences ${ }^{\mathrm{b}}$ & 6.39 & .0001 & 4.48 & 8.30 & \\
\hline Salience of consequences & 5.67 & .005 & 3.33 & 8.01 & \\
\hline Covert sensitization & 4.56 & .016 & 2.43 & 6.68 & \\
\hline Anticipated regret & 4.44 & .018 & 2.34 & 6.55 & \\
\hline $\begin{array}{l}\text { Social and environmental } \\
\text { consequences }\end{array}$ & 4.28 & .041 & 2.05 & 6.51 & .22 \\
\hline $\begin{array}{l}\text { Comparative imagining of } \\
\text { future outcomes }\end{array}$ & 4.17 & .041 & 1.99 & 6.34 & \\
\hline Vicarious reinforcement & $4.00^{\mathrm{a}}$ & .092 & 1.69 & 6.31 & \\
\hline Threat $^{\mathrm{b}}$ & 4.06 & .023 & 2.08 & 6.03 & \\
\hline Pros and cons & $3.67^{\mathrm{a}}$ & .078 & 1.60 & 5.73 & \\
\hline Covert conditioning & 3.50 & .041 & 1.68 & 5.32 & \\
\hline \multicolumn{6}{|l|}{ Reinforcement } \\
\hline Threat $^{\mathrm{b}}$ & 6.78 & .00006 & 4.86 & 8.70 & \\
\hline Self-reward & 5.50 & .006 & 3.20 & 7.80 & \\
\hline Differential reinforcement & 5.33 & .014 & 2.88 & 7.79 & \\
\hline Incentive & 5.39 & .008 & 3.06 & 7.72 & \\
\hline Thinning & 5.28 & .008 & 2.99 & 7.56 & \\
\hline Negative reinforcement & 5.28 & .008 & 3.00 & 7.56 & \\
\hline Shaping & 5.06 & .017 & 2.67 & 7.44 & \\
\hline Counter conditioning & 5.17 & .010 & 2.89 & 7.44 & .28 \\
\hline Discrimination training & 5.06 & .012 & 2.77 & 7.34 & \\
\hline Material reward & 4.89 & .024 & 2.48 & 7.30 & \\
\hline Social reward ${ }^{b}$ & 4.94 & .015 & 2.65 & 7.24 & \\
\hline Non-specific reward & 4.89 & .019 & 2.55 & 7.23 & \\
\hline
\end{tabular}


Table 3. (Continued)

\begin{tabular}{|c|c|c|c|c|c|}
\hline \multirow[b]{2}{*}{ Domain label and associated BCTs } & \multirow{2}{*}{$\begin{array}{c}\text { Mean } \\
\text { confidence } \\
\text { rating }\end{array}$} & \multirow{2}{*}{$\begin{array}{l}\text { Associated } \\
\text { probability }\end{array}$} & \multicolumn{2}{|c|}{$\begin{array}{c}95 \% \\
\text { confidence } \\
\text { intervals }\end{array}$} & \multirow{2}{*}{$\begin{array}{l}\text { Intraclass } \\
\text { correlation } \\
(p<.005)\end{array}$} \\
\hline & & & Lower & Upper & \\
\hline Response cost & 4.94 & .011 & 2.74 & 7.15 & \\
\hline $\begin{array}{l}\text { Anticipation of future } \\
\text { rewards or removal of punishment }\end{array}$ & 4.67 & .022 & 2.40 & 6.94 & \\
\hline Punishment & 4.56 & .025 & 2.30 & 6.81 & \\
\hline Extinction & 4.33 & .018 & 2.28 & 6.39 & \\
\hline Classical conditioning & $3.89^{\mathrm{a}}$ & .078 & 1.69 & 6.09 & \\
\hline \multicolumn{6}{|l|}{ Intentions } \\
\hline Commitment & 4.44 & .022 & 2.14 & 6.75 & .13 \\
\hline Behavioural contract & $3.56^{\mathrm{a}}$ & .064 & 1.45 & 5.66 & \\
\hline \multicolumn{6}{|l|}{ Goals } \\
\hline Goal setting (outcome) & 6.50 & .0007 & 4.13 & 8.87 & \\
\hline Goal setting (behaviour) & 5.50 & .008 & 2.98 & 8.02 & \\
\hline Review of outcome goal(s) & 5.06 & .011 & 2.67 & 7.44 & .23 \\
\hline Review behaviour goals & $4.28^{\mathrm{a}}$ & .057 & 1.82 & 6.74 & \\
\hline $\begin{array}{l}\text { Action planning (including } \\
\text { implementation intentions) }\end{array}$ & 4.39 & .026 & 2.10 & 6.68 & \\
\hline Memory, Attention, and Decision Processes & $\mathrm{N} / \mathrm{A}$ & N/A & $\mathrm{N} / \mathrm{A}$ & $\mathrm{N} / \mathrm{A}$ & .22 \\
\hline \multicolumn{6}{|l|}{ Environmental Context and Resources } \\
\hline Restructuring the physical environment & 6.33 & .001 & 4.03 & 8.64 & \\
\hline Discriminative (learned) cue & 5.33 & .006 & 3.06 & 7.61 & \\
\hline Prompts/cues & 5.17 & .005 & 2.97 & 7.36 & .04 \\
\hline Restructuring the social environment ${ }^{\mathrm{b}}$ & 4.33 & .037 & 2.08 & 6.59 & \\
\hline $\begin{array}{l}\text { Avoidance/changing } \\
\text { exposure to cues for the behaviour }\end{array}$ & $3.67^{\mathrm{a}}$ & .076 & 1.58 & 5.75 & \\
\hline \multicolumn{6}{|l|}{ Social Influences } \\
\hline Social comparison & 6.11 & .001 & 3.86 & 8.36 & \\
\hline Social support or encouragement (general) & 6.11 & .001 & 3.88 & 8.34 & \\
\hline Information about others' approval & 5.72 & .005 & 3.35 & 8.10 & \\
\hline Social support (emotional) ${ }^{\mathrm{b}}$ & 5.50 & .004 & 3.23 & 7.77 & \\
\hline Social support (practical) & 5.00 & .013 & 2.68 & 7.32 & \\
\hline Vicarious reinforcement & 4.89 & .013 & 2.63 & 7.15 & \\
\hline Restructuring the social environment ${ }^{\mathrm{b}}$ & 4.67 & .013 & 2.50 & 6.84 & .19 \\
\hline Modelling or demonstrating the behaviour & 4.44 & .014 & 2.37 & 6.52 & \\
\hline Identification of self as role model & 4.22 & .040 & 2.00 & 6.44 & \\
\hline Social reward ${ }^{\mathrm{b}}$ & $3.89^{\mathrm{a}}$ & .088 & 1.63 & 6.14 & \\
\hline \multicolumn{6}{|l|}{ Emotion } \\
\hline Reduce negative emotions & 5.06 & .014 & 2.71 & 7.40 & \\
\hline Emotional consequences ${ }^{\mathrm{b}}$ & 5.11 & .007 & 2.90 & 7.32 & \\
\hline Self-assessment of affective consequences & 4.78 & .016 & 2.52 & 7.04 & .03 \\
\hline Social support (emotional) ${ }^{\mathrm{b}}$ & $3.94^{\mathrm{a}}$ & .061 & 1.77 & 6.12 & \\
\hline \multicolumn{6}{|l|}{ Behavioural Regulation } \\
\hline Self-monitoring of behaviour & 4.50 & .022 & 2.39 & 6.61 & .32 \\
\hline
\end{tabular}

Notes. BCTs, behaviour change techniques.

Emboldened BCTs are commonly identified BCTs as observed in Michie et al. (2013).

${ }^{a}$ Borderline significant results $p<. I$.

bMapped significantly to multiple domains. 
BCTs in brackets) as follows: Goals (5), Social Influences (4), Environmental Context and Resources (3), Knowledge (2), Reinforcement (2), Skills (1), and Behavioural Regulation (1). The following commonly identified BCTs were not mapped to any of the theoretical domains: Problem solving, Persuasive argument, Discrepancy between current behaviour, Self-monitoring of outcome of behaviour, Monitoring of outcome behaviour by others without feedback, and Pharmacological support.

\section{Comparison of 'bottom-up' and 'top-down' groupings}

The chi-square analyses used for the grouping comparisons did not allow us to include domains that did not have BCTs assigned to them (i.e., where BCTs were not mapped to domains through the DCV process); therefore, the domains of Memory, Attention and Decision Processes and Social/Professional Identity were excluded from these analyses. An additional group of 'No Domain' was included in the 'top-down' groupings and represented cases where BCTs included in the BCT Taxonomy v1 were not assigned to any TDF domain. Therefore, the chi-square analysis was conducted first on $91(7 \times 13)$ possible pairings between the seven higher-order 'bottom-up' sorting strategy groups and the 13 'top-down' groupings and second on $208(16 \times 13)$ possible pairings derived between the original 16 BCT Taxonomy v1 'bottom-up' groupings and the 13 'top-down' groupings.

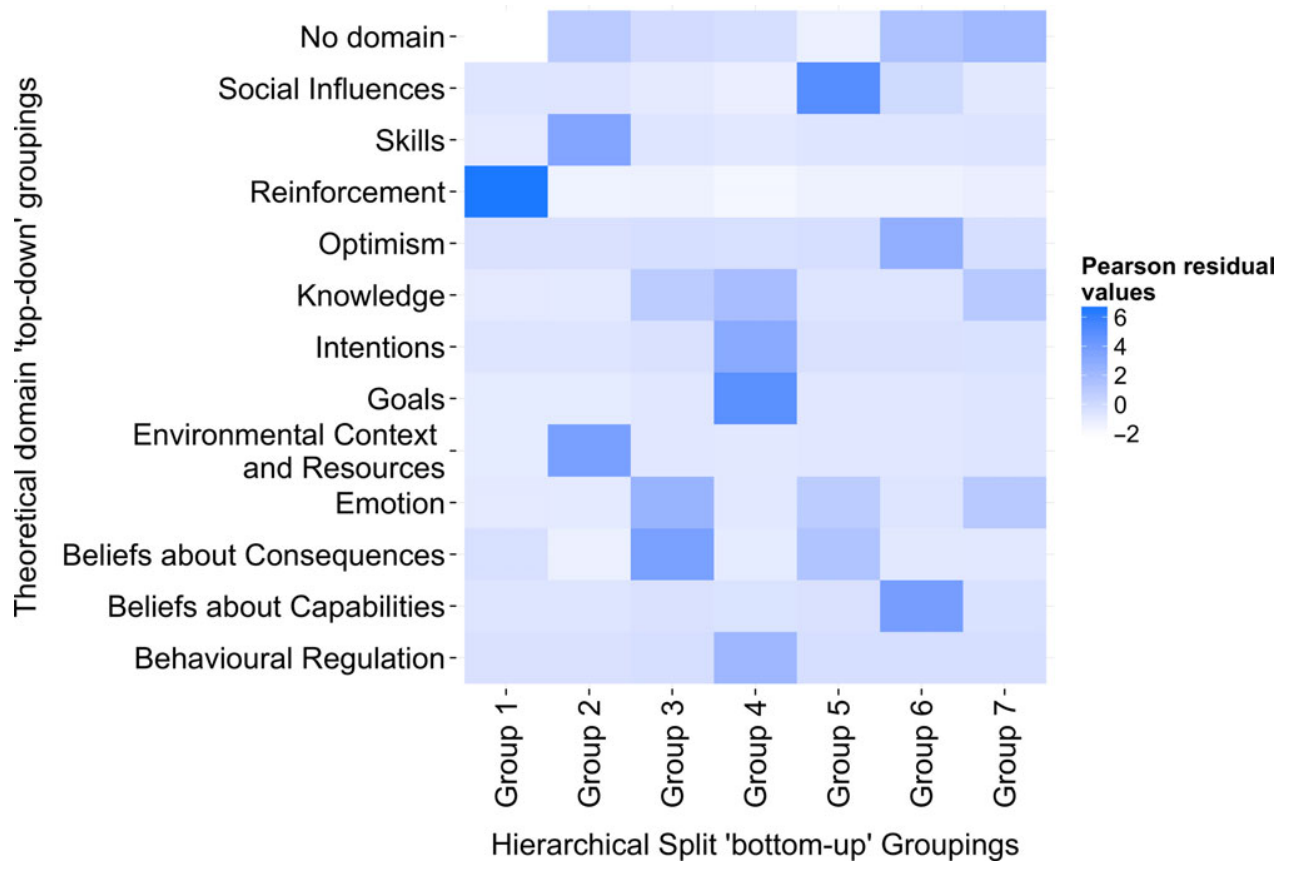

Figure 2. Pearson's residual values for the association between behaviour change technique (BCT) allocation to 'top-down' theoretical domain groupings and the 'bottom-up' higher-order hierarchical split groups. 'No domain' indicates BCTs contained within a 'bottom-up' higher-order group not assigned to any theoretical domains framework domain. Positive Pearson's residual values (darker blue/darker grey in print version) relate to greater overlap than predicted by chance and negative values indicate an overlap less than predicted by chance. 
Table 4. Hierarchical split and TDF domain combinations achieving positive Pearson's residual values for similarities in the assignment of BCTs

\begin{tabular}{llc}
\hline Hierarchical split & TDF domain & \\
Group & Reinforcement & 6.61 \\
\hline Group 1 & Social influences & 5.04 \\
Group 5 & Goals & 4.82 \\
Group 4 & Beliefs about capabilities & 3.80 \\
Group 6 & Environmental context and resources & 3.72 \\
Group 2 & Beliefs about consequences & 3.68 \\
Group 3 & Skills & 3.33 \\
Group 2 & Intentions & 3.05 \\
Group 4 & Optimism & 2.69 \\
Group 6 & Emotion & 2.35 \\
Group 3 & Behavioural regulation & 2.16 \\
Group 4 & No domain & 2.07 \\
Group 7 & Knowledge & 1.77 \\
Group 4 & No domain & 1.49 \\
Group 6 & Beliefs about consequences & 1.40 \\
Group 5 & Emotion & 1.09 \\
Group 7 & Knowledge & 1.09 \\
Group 7 & No domain & 0.94 \\
Group 2 & Emotion & 0.85 \\
Group 5 & Knowledge & 0.85 \\
Group 3 & Social Influences & 0.01 \\
Group 6 & & \\
\hline
\end{tabular}

Notes. BCTs, behaviour change techniques; TDF, Theoretical Domains Framework.

'No domain' indicates BCTs within the hierarchical split groups not assigned to any TDF domains.

Comparison of the BCT groups derived from the higher-order 'bottom-up' sorting strategies, shown in the dendrogram (see Figure 1 and Table S1), and the 'top-down' TDF-derived groups (Table 3$)$ revealed a significant association $\left(\chi^{2}=236.13, p<.001\right)$. Figure 2 shows the level of overlap between each of the group $\times$ TDF pairings within each cell (Pearson's residual value range: -2.10 to 6.61). Twenty-one of the 91 'bottom-up' and 'top-down' TDF Domain combinations showed a greater than expected overlap with positive Pearson's residual values (see Table 4). Only two combinations achieved Pearson's residual values $>5$ : 'Group 1' with 'Reinforcement' (Pearson's residual $=6.61$ ) and 'Group 5' with 'Social Influences' (Pearson's residual $=5.04$ ).

There was also a statistically significant association between the 16 'bottom-up' groupings and the 13 'top-down' groupings $\left(\chi^{2}=437.80, p<.001\right)$. Figure 3 shows the level of overlap between structures; Pearson's residual values range from -1.72 to 6.66 . Thirty-four of the 208 combinations showed greater than expected overlap, achieving positive Pearson's residual values (see Table 5). Six combinations achieved Pearson's residual values $>5$, indicating a comparatively high level of overlap; these combinations were 'Repetition and Substitution' and 'Skills' (Pearson's residual $=6.66$ ), 'Goals and Planning' and 'Goals' (Pearson's residual $=6.41$ ), 'Covert Learning' and 'Beliefs about 'Consequences' (Pearson's residual $=5.76$ ), 'Self-Belief' and 'Beliefs about Capabilities' (Pearson's residual $=5.70)$, 'Scheduled Consequences' and 'Reinforcement' (Pearson's residual $=5.22$ ), and 'Antecedents' and 'Environmental Context and Resources' (Pearson's residual $=5.20$ ). 


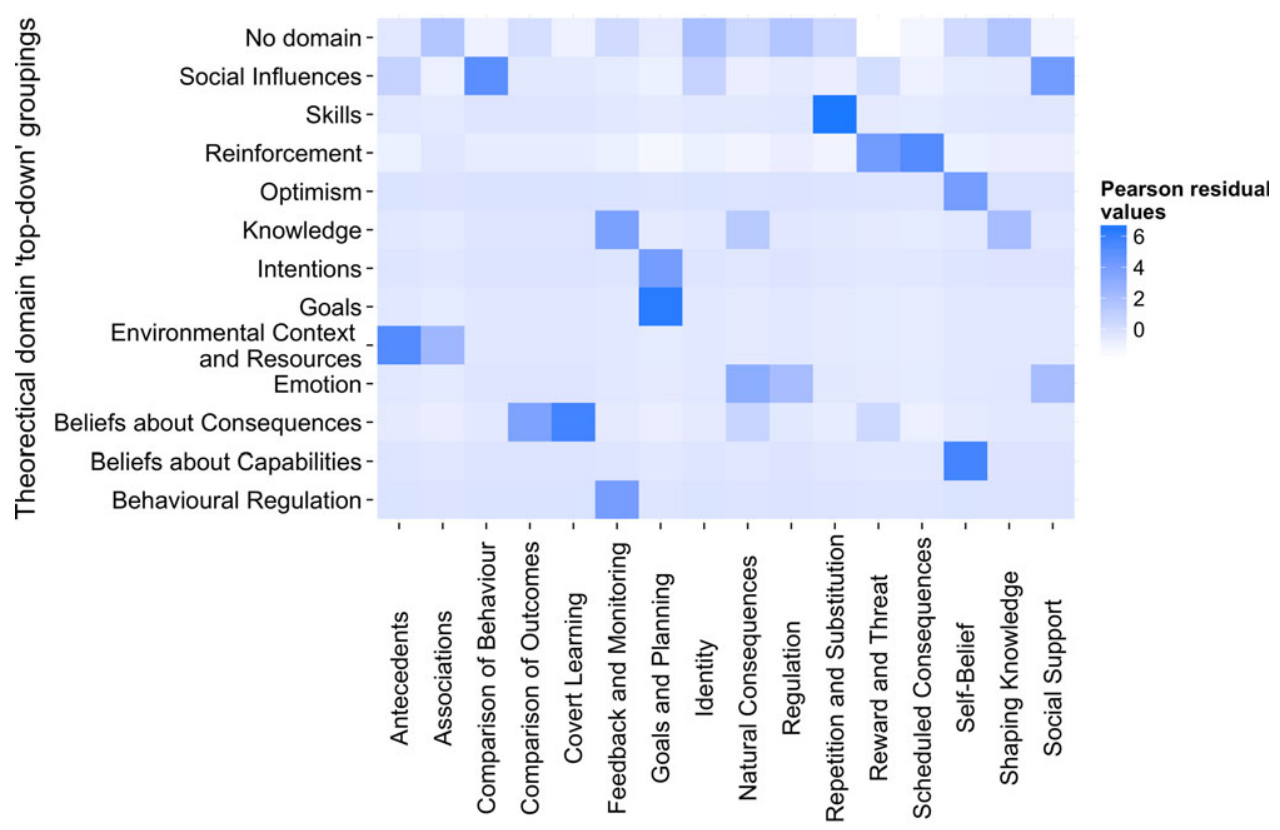

BCT Taxonomy v1 'bottom-up' groupings

Figure 3. Pearson's residual values for the association between behaviour change technique (BCT) allocation to 'top-down' theoretical domain groupings and 'bottom-up' groupings. 'No domain' indicates BCTs within a 'bottom-up' BCT Taxonomy vl grouping not assigned to any domain. Positive Pearson's residual values (darker blue/darker grey in print version) relate to greater overlap than predicted by chance and negative values indicate an overlap less than predicted by chance.

\section{Discussion}

This study examined both the 'bottom-up' hierarchical structure of BCT groupings reported in Taxonomy v1 (Michie et al., 2013) and the 'top-down' grouping of BCTs according to the TDF. It examined the associations and identified overlap in group allocations between these two distinct processes. Examination of the hierarchical structure uncovered a 'higher-order' grouping strategy taken by the behaviour change experts in the 'bottom-up' task, and the dendrogram indicates that some groupings of BCTs within the 16-group solution can be considered as more clearly distinct from others. In contrast, the grouping of BCTs in the 'top-down' sort task has helped illuminate relationships between particular BCTs and theoretical domains and could aid the selection of BCTs in the construction of theory-based interventions. Another key finding was the moderate overlap between the original 16 BCT groupings derived from the 'bottom-up' inductive approach and the 12 groupings from the 'top-down' theoretically driven approach, indicating some common conceptualization of BCTs across these two approaches. These findings may help to further our understanding of the relationships between BCTs and enable researchers to use common BCT group labels to discuss individual, or groups of, BCTs in behaviour change research.

The grouping methods employed in the 'bottom-up' and 'top-down' sort tasks improve on previous attempts to group BCTs in a number of important ways. First, individual groupings of BCTs defined by each expert held equal weight within the final solution and 
Table 5. Taxonomy grouping (bottom-up) and TDF domain (top-down) combinations achieving positive Pearson's residual values for similarities in the assignment of BCTs

\begin{tabular}{|c|c|c|}
\hline Taxonomy grouping & TDF domain & Pearson's residual value \\
\hline Repetition and substitution & Skills & 6.66 \\
\hline Goals and planning & Goals & 6.41 \\
\hline Covert learning & Beliefs about consequences & 5.76 \\
\hline Self-belief & Beliefs about capabilities & 5.70 \\
\hline Scheduled consequences & Reinforcement & 5.22 \\
\hline Antecedents & Environmental context and resources & 5.20 \\
\hline Comparison of behaviour & Social influences & 4.96 \\
\hline Social support & Social influences & 4.14 \\
\hline Reward and threat & Reinforcement & 4.10 \\
\hline Goals and planning & Intentions & 4.05 \\
\hline Feedback and monitoring & Behavioural regulation & 4.03 \\
\hline Self-belief & Optimism & 4.03 \\
\hline Feedback and monitoring & Knowledge & 3.80 \\
\hline Comparison of outcomes & Beliefs about consequences & 3.68 \\
\hline Natural consequences & Emotion & 3.05 \\
\hline Associations & Environmental context and resources & 2.35 \\
\hline Regulation & Emotion & 1.97 \\
\hline Shaping knowledge & Knowledge & 1.97 \\
\hline Social support & Emotion & 1.97 \\
\hline Identity & No domain & 1.83 \\
\hline Regulation & No domain & 1.46 \\
\hline Shaping knowledge & No domain & 1.46 \\
\hline Associations & No domain & 1.45 \\
\hline Natural consequences & Knowledge & 1.25 \\
\hline Antecedents & Social influences & 0.72 \\
\hline Identity & Social influences & 0.72 \\
\hline Natural consequences & Beliefs about consequences & 0.63 \\
\hline Natural consequences & No domain & 0.46 \\
\hline Repetition and substitution & No domain & 0.46 \\
\hline Reward and threat & Beliefs about consequences & 0.37 \\
\hline Feedback and monitoring & No domain & 0.27 \\
\hline Self-belief & No domain & 0.27 \\
\hline Reward and threat & Social influences & 0.12 \\
\hline Comparison of outcomes & No domain & 0.01 \\
\hline
\end{tabular}

Notes. BCTs, behaviour change techniques; TDF, Theoretical Domains Framework.

'No domain' indicates BCTs contained within the hierarchical group not assigned to any TDF domain. Positive Pearson's residual values relate to greater overlap than predicted by chance and negative values indicate an overlap less than predicted by chance.

were aggregated using empirical techniques (HCA in the 'bottom-up' sort task and Discriminant Content Validity methods in the 'top-down' sort task). This is an important development which distinguishes it from earlier work based on consensus approaches using small groups of experts (<5; e.g., Dixon \& Johnston, 2012; Michie, Churchill, et al., 2011; Michie, Hyder, et al., 2011). As a result, the groupings reported here are potentially more robust than those derived using consensus methods.

A second advance was that a comprehensive, cross-behavioural domain taxonomy of BCTs was used, whereas some previous attempts have focused on BCTs relevant to a 
single behavioural domain (e.g., road safety - Geller et al., 1990; smoking cessation Michie, Churchill et al., 2011; weight management - Hardeman, Griffin, Johnston, Kinmonth, \& Wareham, 2000). Thirdly, the BCTs were grouped according to the active ingredients underlying of BCTs, rather than by broad or less well-defined categorizations. In addition to providing 16 groupings, the 'bottom-up' open-sort task yielded systematic empirical estimates of how distinct the groupings are. Examination of this hierarchical structure revealed that BCTs related to reward and threat and those related to cues and cue responses were conceptualized quite distinctly from the other BCTs. The least distinct groups comprised BCTs relating to social support and social comparisons, and the self and identity, suggesting that there is less clarity about the BCTs within these domains. Two further groups of BCTs comprising the BCT Taxonomy v1 groupings of 'Covert Learning' and 'Natural Consequences', and 'Feedback and Monitoring' and 'Goals and Planning', respectively, lay between these most distinct and least distinct groups. The lack of clarity in the least distinct groups may arise for two main reasons. First, there may simply be no agreement about associations between these BCTs. Alternatively, there may be several different formulations about how they are connected. For instance, judgements might be supported by different types of evidence and theoretical frameworks, each of which is internally cohesive. In contrast, BCTs in distinct groupings could potentially share a common mode of action in changing behaviour, whereas BCTs in less distinct groupings may be viewed as having less distinct or more than one mode of action.

The difference in distinctiveness not only has implications for understanding how BCTs are conceptualized by behaviour change experts but also has implications for the practical use of BCT Taxonomy $\mathrm{v} 1$ in behaviour change research. Distinct sets of individual items have been shown to be more easily remembered than less distinct sets (see Baddley, 1966; Polyn, Erlikhman, \& Kahana, 2011; Tulving \& Pearlsto, 1966), and so this variability in distinctiveness may impact on the ability to recall BCTs. This is especially the case where quick reference to BCTs is necessary, for instance when decoding descriptions of interventions or in choosing BCTs to construct a behaviour change intervention. Therefore, in those cases where the groupings are less distinct, adopting additional strategies to aid recall of the groupings may be of particular advantage.

The clusters developed through the 'bottom-up' sort task suggest theoretical thinking. For instance, the first cluster mainly reflects BCTs associated with learning theory, as do those in the third cluster. However, one may ask why these were not grouped together in the first cluster. It would appear that a discrimination is being made between clusters, with the first cluster reflecting BCTs that initiate behaviour change by 'scheduling consequences', whilst the third cluster (labelled as 'Repetition and Substitution') deals with the maintenance of behaviour change including maintenance in the face of competing behaviours. Thus, the clustering may not only reflect current explicit theorizing but also indicate where developments are being made.

The 'top-down' mapping of BCTs to theoretical domains advances the limited consensus methods used by Michie et al. (2008) by using an improved BCT taxonomy, an empirically validated theory domains framework and a larger number of respondents. In this 'top-down' task, 69/87 BCTs were reliably allocated to one or more of the TDF domains with a further 12 BCTs having borderline statistical significance. The majority of BCTs (37) were allocated to three domains which also had high confidence ratings and ICCs: 'Beliefs about Consequences', 'Reinforcement', and 'Social Influences'. This suggests that these are the theoretical domains where there is the greatest number of agreed methods for bringing about change. Other domains also showed high agreement but had fewer associated BCTs - 'Behavioural regulation' had only one assigned BCT but 
achieved good agreement, whilst 'Goals' had five BCTs assigned with good agreement. In designing interventions, it may be more important to have a few agreed BCTs than to have a large choice of BCTs available to change a given theoretical determinant of behaviour. Further evidence is required to ensure that these 'agreed' BCTs do in fact achieve behaviour change by changing the proposed theoretical domain. For the two theoretical domains where no BCTs were reliably assigned, there would appear to be no shared, or recognized, way of changing them.

Most of the commonly used BCTs were associated with a theoretical domain. Of the 24 most frequently identified BCTs in Michie et al. (2013), 17 were clearly grouped into one of the 14 domains; the remaining seven BCTs were not reliably allocated to any domain even though they could be identified reliably in the intervention descriptions. This finding suggests that these BCTs may have evolved from several different theoretical approaches and therefore may be less associated with a particular theoretical domain.

\section{Comparison of open- and closed-sort tasks}

Six of the open-sort tasks groupings - 'Repetition and Substitution, 'Goals and Planning', 'Covert Learning', 'Self-Belief', 'Scheduled Consequences', and 'Antecedents' - showed a high level of overlap with the six of the TDF groupings, suggesting that experts may have sorted BCTs by theoretical constructs or domains (implicitly or explicitly) across both tasks. This is supported by the fact that both groups reported high levels of expertise in relation to behaviour change theory.

By contrast, there were only two similarly strong overlaps between the higher-order groupings and the TDF groups, indicating that the relationship between higher-order sorting strategies and theoretically derived groups is not strong. It would appear that the lower level groupings are more in line with the theoretical frameworks than the empirically higher-order groupings, suggesting that the higher level of sorting shared by respondents does not align as well with the theoretical domains. Perhaps the higher-order groupings of BCTs depended on considerations other than theory, for example target populations or behaviours.

\section{Potential limitations and future research}

One potential limitation of the present study is that it is likely that experts' judgements are influenced by specific theoretical approaches - or cues from particular words especially those associated with theories that have a longer history. However, the approaches taken in the grouping methodology helped to overcome this potential bias in two ways. First, the use of the word 'theory' was avoided in the open-sort task instructions to prevent priming the use of theory to sort BCTs. Second, the clustering and Discriminant Content Validity techniques used allowed for the aggregation of sort task responses across participants diluting any individual viewpoint. Such an approach is less prone to bias from individual responses and therefore provides a more balanced view of how experts, as a whole, conceptualize BCTs. Nevertheless, examining the variability of approaches in judgements from different kinds of expert might be of interest in future research.

The next step for this line of research is to evaluate the extent to which these groupings facilitate the usability of the taxonomy. It may be that different groupings may be useful for different tasks (e.g., identifying BCTs vs. designing interventions) and/or be beneficial to different users in different contexts. It may be that for those applying BCTs to designing or 
specifying interventions without reference to theory, the open-sort groupings may be of more benefit as all of the BCTs were incorporated into the groups. On the other hand, the closed-sort grouping of BCTs is likely to be more useful for those who are seeking a theoretical base for coding and designing interventions. Further work will be necessary to investigate the replicability and utility of these groupings, as well as their theoretical basis. As more evidence is gained from the application of BCT Taxonomy v1, the BCT groupings may be modified to incorporate links between BCTs that are commonly used together in research practice and/or to reflect the 'common mechanisms of action'.

\section{Acknowledgements}

We would like to thank the BCT Taxonomy co-investigators Charles Abraham, Jill Francis, Wendy Hardeman, Martin Eccles, and Caroline Wood for their comments and help in relation to this work. This work was supported through a Medical Research Council Health Service Research Collaboration grant (ref. G0901474).

\section{References}

Abraham, C., Good, A., Warren, M. R., Huedo-Medina, T., \& Johnson, B. (2011). Developing and testing a SHARP taxonomy of behavior change techniques included in condom promotion interventions. Psychology and Health, 26(Suppl. 2), 299. doi:10.1080/17437199.2012.654964

Abraham, C., \& Michie, S. (2008). A taxonomy of behavior change techniques used in interventions. Health Psychology: Official Journal of the Division of Health Psychology, American Psychological Association, 27, 379-387. doi:10.1037/0278-6133.27.3.379

Amemori, M., Korhonen, T., Kinnunen, T., Michie, S., \& Murtomaa, H. (2011). Enhancing implementation of tobacco use prevention and cessation counselling guideline among dental providers: A cluster randomised controlled trial. Implementation Science, 6 (1), 13. doi:10. $1186 / 1748-5908-6-13$

Araújo-Soares, V., McIntyre, T., Maclennan, G., \& Sniehotta, F. F. (2009). Development and exploratory cluster-randomised opportunistic trial of a theory-based intervention to enhance physical activity among adolescents. Psychology \& Health, 24, 805-822. doi:10.1080/ 08870440802040707

Baddley, A. (1966). Short-term memory for word sequences as a function of acoustic, semantic and formal similarity. Quarterly Journal of Experimental Psychology, 18, 362-365. doi:10.1080/ 14640746608400055

Beenstock, J., Sniehotta, F. F., White, M., Bell, R., Milne, E., \& Araujo-Soares, V. (2012). What helps and hinders midwives in engaging with pregnant women about stop smoking? A cross-sectional survey of percieved implementation difficulties among midwives in the North East of England. Implementation Science, 7, 36. doi:10.1186/1748-5908-7-36

Boutron, I., Moher, D., Altman, D. G., Schulz, K. F., \& Ravaud, P. (2008). Extending the CONSORT statement to randomized trials of nonpharmacologic treatment: Explanation and elaboration. Annals of Internal Medicine, 148, 295-309. doi:10.7326/0003-4819-148-4-200802190-00008

Bussières, A. E., Patey, A. M., Francis, J. J., Sales, A. E., \& Grimshaw, J. M. (2012). Identifying factors likely to influence compliance with diagnostic imaging guideline recommendations for spine disorders among chiropractors in North America: A focus group study using the Theoretical Domains Framework. Implementation Science, 7 (1), 1-11. doi:10.1186/1748-5908-7-82

Cane, J., O'Connor, D., \& Michie, S. (2012). Validation of the theoretical domains framework for use in behaviour change and implementation research. Implementation Science, 7 (1), 37. doi:10. 1186/1748-5908-7-37

Carmines, E. G., \& Zeller, R. A. (1979). Reliability and validity assessment. London, UK: SAGE. 
Chadwick, P., \& Benelam, B. (2013). Using behaviour change taxonomies to improve service delivery - a workshop with nutritionists and dietitians. Nutrition Bulletin, 38, 108-111. doi:10. 1111/nbu.12013

Craig, P., Dieppe, P., Macintyre, S., Michie, S., Nazareth, I., \& Petticrew, M. (2008). Developing and evaluating complex interventions: The new Medical Research Council guidance. British Medical Journal, 337, 979-983. doi:10.1136/bmj.a1655

Davies, P., Walker, A. E., \& Grimshaw, J. (2010). A systematic review of the use of theory in the design and implementation strategies and interpretation of the results of rigorous evaluations. Implementation Science, 5, 1-6. doi:10.1186/1748-5908-5-14

Dixon, D., \& Johnston, M. (2012). Health Behavior Change Competency Framework: Competences to deliver interventions to change lifestyle behaviors that affect bealth. Presented at the Scottish Government, Edinburgh.

Dixon, D., Johnston, M., McQueen, M., \& Court-Brown, C. (2008). The Disabilities of the Arm, Shoulder and Hand Questionnaire (DASH) can measure the impairment, activity limitations and participation restriction constructs from the International Classification of Functioning, Disability, and Health (ICF). BMC Musculoskeletal Disorders, 9, 114. doi:10.1186/ 1471-2474-9-114

Dixon, D., Pollard, B., \& Johnston, M. (2007). What does the chronic pain grade questionnaire measure? Pain, 130, 249-253. doi:10.1016/j.pain.2006.12.004

Dombrowski, S. U., Sniehotta, F. F., Avenell, A., Johnston, M., MacLennan, G., \& Araújo-Soares, V. (2012). Identifying active ingredients in complex behavioural interventions for obese adults with obesity-related co-morbidities or additional risk factors for co-morbidities: A systematic review. Health Psychology Review, 6 (1), 7-32. doi:10.1080/17437199.2010.513298

Dyson, J., Lawton, R., Jackson, C., \& Cheater, F. (2011). Does the use of a theoretical approach tell us more about hand hygiene behaviour? The barriers and levers to hand hygiene. Journal of Infection Prevention, 12 (1), 17-24. doi:10.1177/1757177410384300

Francis, J. J., Stockton, C., Eccles, M. P., Johnston, M., Cuthbertson, B. H., Grimshaw, J. M., ... Stanworth, S. J. (2009). Evidence-based selection of theories for designing behaviour change interventions: Using methods based on theoretical construct domains to understand clinicians' blood transfusion behaviour. British Journal Health Psychology, 14, 625-646. doi:10.1348/ $135910708 \times 397025$

Francis, J. J., Tinmouth, A., Stanworth, S. J., Grimshaw, J. M., Johnston, M., Hyde, C., ... Eccles, M. (2009). Using theories of behaviour to understand transfusion prescribing in three clinical contexts in two countries: Development work for an implementation trial. Implementation Science, 4 (1), 70. doi:10.1186/1748-5908-4-70

Free, C., Phillips, G., Watson, L., Galli, L., Felix, L., Edwards, P., ... Haines, A. (2013). The effectiveness of mobile-health technologies to improve health care service delivery processes: A systematic review and meta-analysis. PLOS Med, 10 (1), e1001363. doi:10.1371/journal.pmed. 1001363

French, S. D., Green, S. E., O’Connor, D. A., McKenzie, J. E., Francis, J. J., Michie, S., .. Grimshaw, J. M. (2011). Developing theory-informed behaviour change interventions to implement evidence into practice: A systematic approach using the Theoretical Domains Framework. Implementation Science, 7, 38. doi:10.1186/1748-5908-7-38

Geller, E. S., Berry, T. D., Ludwig, T. D., Evans, R. E., Gilmore, M. R., \& Clarke, S. W. (1990). A conceptual framework for developing and evaluating behavior change interventions for injury control. Health Education Research, 5, 125-137. doi:10.1093/her/5.2.125

Hardeman, W., Griffin, S., Johnston, M., Kinmonth, A. L., \& Wareham, N. J. (2000). Interventions to prevent weight gain: A systematic review of psychological models and behaviour change methods. International Journal of Obesity, 24, 131-143. doi:10.1038/sj.ijo.0801100

Hobbs, L., Campbell, R., Hildon, Z., \& Michie, S. (2011). Behaviour change theories across Psychology, Sociology, Anthropology and Economics: A Systematic Review. Psychology and Health, 26(Suppl. 1), 31. 
Hochberg, Y. (1988). A sharper Bonferroni procedure for multiple tests of significance. Biometrika, 75, 800-802. doi:10.1093/biomet/75.4.800

Ivers, N., Jamtvedt, G., Flottorp, S., Young, J. M., Odgaard-Jensen, J., French, S. D., . . Oxman, A. D. (2012). Audit and feedback: Effects on professional practice and healthcare outcomes. Cochrane Database of Systematic Reviews (Online), 6, CD000259. doi:10.1002/14651858.CD000259.pub3

Lorencatto, F., West, R., \& Michie, S. (2012). Specifying evidence-based behavior change techniques to aid smoking cessation in pregnancy. Nicotine E Tobacco Research: Official Journal of the Society for Research on Nicotine and Tobacco, 14, 1019-1026. doi:10.1093/ntr/ntr324

Lynn, M. R. (1986). Determination and quantification of content validity. Nursing Research, 35, 382-385. doi:1097/00006199-198611000-00017

McKenzie, J. E., French, S. D., O'Connor, D. A., Grimshaw, J. M., Mortimer, D., Michie, S., .. Green, S. E. (2008). IMPLEmenting a clinical practice guideline for acute low back pain evidence-based manageMENT in general practice (IMPLEMENT): Cluster randomised controlled trial study protocol. Implementation Science, 3, 11. doi:10.1186/1748-5908-3-11

McKenzie, J. E., O'Connor, D., Page, M. J., Mortimer, D., French, S. D., Walker, B. F., . . Green, S. E. (2010). Improving the care for people with acute low-back pain by allied health professionals (the ALIGN trial): A cluster randomised trial protocol. Implementation Science, 5, 86. doi:10. $1186 / 1748-5908-5-86$

Michie, S., Abraham, C., Eccles, M. P., Francis, J. J., Hardeman, W., \& Johnston, M. (2011). Strengthening evaluation and implementation by specifying components of behaviour change interventions: A study protocol. Implementation Science, 6 (1), 10. doi:10.1186/1748-5908-6-10

Michie, S., Abraham, C., Whittington, C., McAteer, J., \& Gupta, S. (2009). Effective techniques in healthy eating and physical activity interventions: A meta-regression. Health Psychology: Official Journal of the Division of Health Psychology, American Psychological Association, 28, 690-701. doi:10.1037/a0016136

Michie, S., Churchill, S., \& West, R. (2011). Identifying evidence-based competences required to deliver behavioral support for smoking cessation. Annals of Behavioral Medicine, 41, 59-70. doi:10.1007/s12160-010-9235-z

Michie, S., Free, C., \& West, R. (2012). Characterising the "Txt2Stop" smoking cessation text messaging intervention in terms of behaviour change techniques. Journal of Smoking Cessation, 7 (1), 55-60. doi:10.1017/jsc.2012.12

Michie, S., Hyder, N., Walia, A., \& West, R. (2011). Development of a taxonomy of behaviour change techniques used in individual behavioural support for smoking cessation. Addictive Behaviors, 36, 315-319. doi:10.1016/j.addbeh.2010.11.016

Michie, S., \& Johnston, M. (2012). Theories and techniques of behaviour change: Developing a cumulative science of behaviour change. Health Psychology Review, 6 (1), 1-6. doi:10.1080/ 17437199.2012 .654964

Michie, S., Johnston, M. (2013). Behavior change techniques. In M. D. Gellman \& J. R. Turner (Eds.), Encyclopaedia of behavioral medicine (pp. 182-187). New York: Springer.

Michie, S., Johnston, M., Abraham, C., Francis, J., Hardeman, W., Eccles, M., \& Cane, J. (2012). An 87-item taxonomy of Behavior Change Techniques: Building an international consensus for the reporting of behavior change interventions. Proceedings paper. Retrieved from abstracts. bps.org.uk/index.cfm?\&ResultsType=Abstracts\&ResultSet_ID=8288\&FormDisplayMode=view\& frmShowSelected $=$ true $\&$ localAction $=$ details

Michie, S., Johnston, M., Abraham, C., Lawton, R., Parker, D., \& Walker, A. (2005). Making psychological theory useful for implementing evidence based practice: A consensus approach. Quality \& Safety in Health Care, 14, 26-33. doi:10.1136/qshc.2004.011155

Michie, S., Johnston, M., Francis, J., Hardeman, W., \& Eccles, M. (2008). From theory to intervention: Mapping theoretically derived behavioural determinants to behaviour change techniques. Applied Psychology, 57, 660-680. doi:10.1111/j.1464-0597.2008.00341.x

Michie, S., Richardson, M., Johnston, M., Abraham, C., Francis, J., Hardeman, W., ... Wood, C. (2013). The behavior change technique taxonomy (v1) of 93 hierarchically clustered 
techniques: Building an international consensus for the reporting of behavior change interventions. Annals of Behavioral Medicine, 46 (1), 81-95. doi:10.1007/s12160-013-9486-6

Michie, S., Whittington, C., Hamoudi, Z., Zarnani, F., Tober, G., \& West, R. (2012). Identification of behaviour change techniques to reduce excessive alcohol consumption. Addiction (Abingdon, England), 107, 1431-1440. doi:10.1111/j.1360-0443.2012.03845.x

Mokdad, A. H., Marks, J. S., Stroup, D. F., \& Gerberding, J. L. (2004). Actual causes of death in the United States, 2000. Journal of the American Medical Association, 291, 1238-1245. doi:10. 1001/jama.291.10.1238

Parkin, D. M., Boyd, L., \& Walker, L. C. (2011). 16. The fraction of cancer attributable to lifestyle and environmental factors in the UK in 2010. Britisb Journal of Cancer, 105, S77-S81. doi:10.1038/ bjc. 2011.489

Patey, A. M., Islam, R., Francis, J. J., Bryson, G. L., \& Grimshaw, J. M. (2012). Anesthesiologists' and surgeons' perceptions about routine pre-operative testing in low-risk patients: Application of the Theoretical Domains Framework (TDF) to identify factors that influence physicians' decisions to order pre-operative tests. Implementation Science, 7 (1), 1-13. doi:10.1186/17485908-7-52

Polyn, S. M., Erlikhman, G., \& Kahana, M. J. (2011). Semantic cuing and the scale insensitivity of recency and contiguity. Journal of Experimental Psychology. Learning Memory and Cognition, 37, 766-775. doi:10.1037/a0022475

R Development Core Team (2008). R: A language and environment for statistical computing. Vienna, Austria: R Foundation for Statistical Computing. ISBN 3-900051-07-0. Retrieved from http://www.R-project.org

Taylor, N., Conner, M., \& Lawton, R. (2012). The impact of theory on the effectiveness of worksite physical activity interventions: A meta-analysis and meta-regression. Health Psychology Review, 6 (1), 33-73. doi:10.1080/17437199.2010.533441

Tulving, E., \& Pearlsto, Z. (1966). Availability versus accessibility of information in memory for words. Journal of Verbal Learning and Verbal Behavior, 5, 381-391. doi:10/1016/S0022-5371 (66)80048-4

Waltz, C. W., Strickland, O. L., \& Lenz, E. R. (1991). Measurement in nursing research. Philadelphia, PA: F.A.Davis Co. doi:10.1002/nur.4770080120

Webb, T. L., Joseph, J., Yardley, L., \& Michie, S. (2010). Using the internet to promote health behavior change: A systematic review and meta-analysis of the impact of theoretical basis, use of behavior change techniques, and mode of delivery on efficacy. Journal of Medical Internet Research, 12 (1), e4. doi:10.2196/jmir.1376

West, R., Walia, A., Hyder, N., Shahab, L., \& Michie, S. (2010). Behavior change techniques used by the English Stop Smoking Services and their associations with short-term quit outcomes. Nicotine \& Tobacco Research, 12, 742-747. doi:10.1093/ntr/ntq074

Received 4 November 2013; revised version received I April 20/4

\section{Supporting Information}

The following supporting information may be found in the online edition of the article:

Appendix S1. Behaviour Change Technique Taxonomy used in the sort tasks.

Appendix S2. Two - Theoretical Domains Framework domain labels and definitions.

Appendix S3. Three - Closed 'top-down' sort task instructions.

Table S1. Comparison of BCT Taxonomy v1 'bottom-up' grouping solution and 'top-down' 14 TDF-derived groupings. 